Uniformly Bounded Information and Social Choice

Donald E. Campbell

College of William and Mary

Jerry S. Kelly

Syracuse University

College of William and Mary

Department of Economics

Working Paper Number 49

January 2007 
COLLEGE OF WILLIAM AND MARY

DEPARTMENT OF ECONOMICS

WORKING PAPER \# 49

January 2007

\title{
Uniformly Bounded Information and Social Choice
}

\begin{abstract}
The set of alternatives is infinite. If social welfare function $\mathrm{f}$ satisfies the Pareto criterion and there is a positive number $\beta$ such that each pair of alternatives can be socially ordered without having to consult individual preference over a set with more than $\beta$ alternatives then there are arbitrarily large finite subsets of $\mathrm{X}$ within which $\mathrm{f}$ is dictatorial when individual preferences are restricted to a rich subset of profiles.
\end{abstract}

JEL Codes: D70, D71

Keywords: chromatic graphs, generalized IIA, social welfare function

Donald E. Campbell

Department of Economics

College of William and Mary

Williamsburg, VA 23187-8795

decamp@wm.edu
Jerry S. Kelly

Department of Economics

Syracuse University

Syracuse, NY 13245-1090

jskelly@maxwell.syr.edu 


\section{Uniformly Bounded Information and Social Choice}

K. J. Arrow's general specification of the social choice problem was criticized from the beginning for employing a very restrictive informational assumption; namely, arbitrary alternatives $\mathrm{x}$ and $\mathrm{y}$ can be socially ordered without reference to individual preference comparisons outside of the set $\{x, y\} .{ }^{1}$ At the other extreme is the global Borda rule and most of its positional relatives. For each of those social welfare functions a pair of alternatives typically cannot be socially ordered without obtaining information about each individual's preference relation over the entire set X. Merely identifying the set X of feasible alternatives is prohibitively costly in most situations, let alone the cost of gathering information about individual preferences over a large set. Few treatments of social choice lie between the two extremes. ${ }^{2}$ (If $\mathrm{X}$ is a set of potential candidates for national political office, before we get very far in listing the individuals who meet the eligibility criteria one of them will die. If $\mathrm{X}$ is a set of feasible allocations in an economic context, then the process of identifying $\mathrm{X}$ will consume resources and hence change the feasible set. Even the passage of time will result in lost opportunities for capital formation and consequently a different feasible set.)

This paper employs a less demanding informational assumption than the global Borda rule, but one that allows for much more individual preference information to be used to socially order a pair of alternatives than does IIA, Arrow's independence of irrelevant alternatives condition. We replace IIA with the requirement that there be a positive number $\beta$ such that for each pair $\{x, y\}$ of alternatives there is a $\beta$-element set $Y$ such that $x$ and $y$ can be socially ordered without consulting individual preferences outside of Y. (The set Y will be different for different pairs.) Whatever the individual preferences, only the preference comparisons involving members of $Y$ can affect the social ordering of $x$ and $y$. We emphasize that $\beta$ can be any positive integer, however large, but there must be a single $\beta$ that suffices for all pairs of alternatives. By assuming an infinite set of alternatives we can create a model in which departures from IIA are non-trivial but "small".

1. Baumol (1952) is an early instance and Cowell (2006), page 231, is a recent one. Osborne (1976) is also noteworthy, as is Sen (1986).

2. Important exceptions include Fleurbaey (2003), Fleurbaey and Maniquet (2005), and Fleurbaey, Suzumura, and Tadenuma (2005). See also Campbell and Kelly (2000 and 2007). 
For a standard domain, we show that every social welfare function satisfying the Pareto criterion and the boundedness condition is sub-dictatorial in the following sense: For every positive integer $\alpha \geq 3$ there is an infinite collection of finite subsets $\mathrm{A}$ of $\mathrm{X}$, each with $\alpha$ members, such that within the subfamily of profiles for which every individual ranks every member of A above every member of X\A, and the members of $\mathrm{X} \backslash \mathrm{A}$ are individually ranked in a fixed way (but not necessarily in the same way for each individual), the social ordering of the members of $\mathrm{A}$ is dictatorial. This paper actually proves a strong version of this claim.

\section{Preliminaries}

The infinite set $\mathrm{X}$ is a universal set of outcomes and $\mathrm{N}$ is the (finite) set of individuals, the society. A weak ordering on $\mathrm{X}$ is a complete and transitive binary relation on $\mathrm{X}$, and we let $\mathrm{W}(\mathrm{X})$ denote the set of all weak orderings on $\mathrm{X}$. For $\mathrm{R} \in \mathrm{W}(\mathrm{X})$ and $\mathrm{Y} \subset \mathrm{X}$ let $\mathrm{R} \mid \mathrm{Y}$ denote the relation $\mathrm{R} \cap \mathrm{Y} \times \mathrm{Y}$ on $\mathrm{Y}$, the restriction of $\mathrm{R}$ to $\mathrm{Y}$. We let $\mathrm{L}(\mathrm{X})$ represent the set of all strong orderings on $\mathrm{X}$. (We say that $\mathrm{R} \in \mathrm{W}(\mathrm{X}$ ) is strong if it is antisymmetric, which means that $(\mathrm{x}, \mathrm{y})$ and $(\mathrm{y}, \mathrm{x})$ both belong to $\mathrm{R}$ if and only if $\mathrm{x}=\mathrm{y}$.)

A profile $\mathrm{p}$ is a member of $\mathrm{W}(\mathrm{X})^{\mathrm{N}}$, and it assigns the ordering $\mathrm{p}(\mathrm{i})$ to individual i. A preference domain is a nonempty subset $\rho$ of $\mathrm{W}(\mathrm{X})^{\mathrm{N}}$. For each subset $\mathrm{Y}$ of $\mathrm{X}$ and each profile $\mathrm{p}$ in $\rho$ let $\mathrm{p} \mid \mathrm{Y}$ denote the restriction of profile $p \in \rho$ to $Y$. That is, $p \mid Y$ represents the function $q \in W(Y)^{N}$ satisfying $q(i)=$ $\mathrm{p}(\mathrm{i}) \mid \mathrm{Y}$ for all $\mathrm{i} \in \mathrm{N}$. Given a domain $\rho$ for $\mathrm{X}$ and a subset $\mathrm{Y}$ of $\mathrm{X}$ we let $\rho \mid \mathrm{Y}$ represent the set $\{\mathrm{p} \mid \mathrm{Y}: \mathrm{p} \in \mathcal{\rho}\}$.

A social welfare function for domain $\rho$ is a function $f$ that specifies a binary relation $f(p)$ on $X$ for each $\mathrm{p} \in \mathcal{P}$. Given a profile $\mathrm{p}$, we let $\mathrm{x} \succ_{\mathrm{i}}^{\mathrm{p}} \mathrm{y}$ indicate that individual $\mathrm{i}$ either prefers $\mathrm{x}$ to $\mathrm{y}$ or is indifferent between $x$ and $y$ at profile $p$. Of course, $x \succ_{i}^{p} y$ means that i strictly prefers $x$ to $y$ according to $p(i)$. If $f: \rho \rightarrow W(X)$, which means that $f$ is a social welfare function with domain $\rho$, then $x \succeq_{f(p)} y$ means that $\mathrm{x}$ ranks at least as high as $\mathrm{y}$ in the social ordering $\mathrm{f}(\mathrm{p})$, and $\mathrm{x} \succ_{\mathrm{f}(\mathrm{p})} \mathrm{y}$ means that $\mathrm{x}$ ranks strictly higher

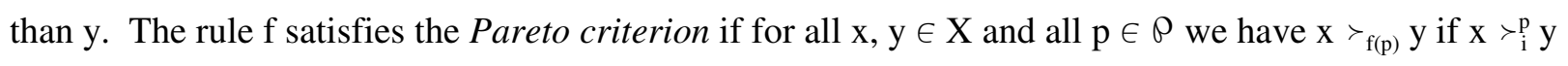
for all $\mathrm{i} \in \mathrm{N}$, and $\mathrm{f}$ is anonymous if $\mathrm{f}(\mathrm{p})=\mathrm{f}(\mathrm{q})$ whenever $\mathrm{q}$ can be obtained from $\mathrm{p}$ by replacing $\mathrm{p}(\mathrm{i})$ with $\mathrm{p}(\mathrm{j})$ and $\mathrm{p}(\mathrm{j}$ ) with $\mathrm{p}(\mathrm{i})$ for arbitrary $\mathrm{i}$ and $\mathrm{j}$ in $\mathrm{N}$. We say that $\mathrm{f}$ satisfies IIA if for all $\mathrm{x}$ and $\mathrm{y}$, and any two admissible profiles $\mathrm{p}$ and $\mathrm{q}$, we have $\mathrm{f}(\mathrm{p})|\{\mathrm{x}, \mathrm{y}\}=\mathrm{f}(\mathrm{q})|\{\mathrm{x}, \mathrm{y}\}$ if $\mathrm{p}|\{\mathrm{x}, \mathrm{y}\}=\mathrm{q}|\{\mathrm{x}, \mathrm{y}\}$.

We do not assume IIA (although it will cast its shadow), and hence the Pareto criterion does not imply dictatorship. However, our restrictions on the social welfare function will be shown to imply that it has elements of dictatorship — specifically, it belongs to the following family of sub-dictatorial rules. 
Informally, a rule is sub-dictatorial if there is an infinite number of sets of arbitrary cardinality within which one person's strict preference determines strict social preference over an infinite subset of the domain.

\section{Sub-dictatorial social welfare function:}

If $\mathrm{f}$ has domain $\rho$ we say that it is sub-dictatorial if for every positive integer $\alpha \geq 3$ there is an infinite collection $\boldsymbol{A}$ of subsets of $\mathrm{X}$ with the following two properties:

1. $\quad$ Each member of $\boldsymbol{A}$ has exactly $\alpha$ members

2. For each $\mathrm{A} \in \boldsymbol{A}$ there is a disjoint and finite subset $\mathrm{B}$ of $\mathrm{X}$ such that for every subset $\mathrm{Q}^{\prime}$ of $\rho$ satisfying

(i) $\quad \rho^{\prime} \mid \mathrm{A}$ equals $\mathrm{L}(\mathrm{A})^{\mathrm{N}}$ or $\mathrm{W}(\mathrm{A})^{\mathrm{N}}$,

(ii) For all $\mathrm{a} \in \mathrm{A}$ and $\mathrm{b} \in \mathrm{B}$ we have $\mathrm{p}|\{\mathrm{a}, \mathrm{b}\}=\mathrm{q}|\{\mathrm{a}, \mathrm{b}\}$ for all $\mathrm{p}$ and $\mathrm{q} \in \mathrm{\rho}^{\prime}$

(iii) $\mathrm{p}|\mathrm{B}=\mathrm{q}| \mathrm{B}$ for all $\mathrm{p}, \mathrm{q} \in \mathrm{\rho}^{\prime}$

there is an individual $j \in N$ such that, for all $x$ and $y$ in $A, x \succ_{j}^{p} y$ implies $x \succ_{f(p)} y$ for every profile $\mathrm{p} \in \mathrm{\rho}^{\prime}$.

Properties (i) and (ii) imply that at any profile in $\rho^{\prime}$ a member of $X \backslash A$ cannot be indifferent to any member of A for any individual, nor can there be one member of A ranking above and another ranking below some member of $\mathrm{B} \backslash \mathrm{A}$ in the preference ordering of any individual. Suppose to the contrary we had $\mathrm{a} \succ_{\mathrm{i}}^{\mathrm{p}} \mathrm{b} \succ_{\mathrm{i}}^{\mathrm{p}} \mathrm{a}^{\prime}$ for $\mathrm{a}$ and $\mathrm{a}^{\prime}$ in $\mathrm{A}$ and $\mathrm{b}$ in $\mathrm{B}$. By (i) there is another profile in $\mathrm{O}^{\prime}$ at which individual $\mathrm{i}$ ranks $a^{\prime}$ above or indifferent to $a$. That would force a change in the individual's ranking of $a$ and $b$ or $a^{\prime}$ and $b$, contradicting (ii). For an example of $\rho^{\prime}$ satisfying (i) - (iii) set $\rho=L(X)^{N}$ and let $\rho^{\prime}$ be the subset of $\rho$ within which every alternative in A ranks above every alternative in X/A for every individual, with the members of A be arbitrarily ordered with respect to each other, and with each individual's ordering of the members of B fixed with respect to each other.

Given $\beta$, the proof of our theorem actually gives an upper bound on the cardinality of B that is a constant multiple of $\alpha$.

When we refer to a pair of alternatives from now on we will mean two distinct members of $X$. If the restriction of arbitrary profile $\mathrm{p}$ to set $\mathrm{Y}$ contains all the information that is needed to social order a pair of alternatives we say that $\mathrm{Y}$ is sufficient for that pair. 


\section{Sufficient set}

Given social welfare function $f$ on domain $\rho$ the subset $Y$ of $X$ is sufficient for the pair of alternatives $\{x, y\}$ if for all $p$ and $q$ in $\rho$ such that $p|Y=q| Y$ we have $f(p)|\{x, y\}=f(q)|\{x, y\}$.

If $\mathrm{Y}$ is sufficient for $\{\mathrm{x}, \mathrm{y}\}$ then individual preferences restricted to $\mathrm{Y}$ provide enough information to socially order $\mathrm{x}$ and $\mathrm{y}$. $\mathrm{Y}$ itself may contain a proper subset that is also sufficient for $\{\mathrm{x}, \mathrm{y}\}$. If there is a smallest sufficient set then we refer to it as the relevant set for the pair in question.

\section{Relevant set:}

Given a social welfare function $\mathrm{f}$ on domain $\rho$ we say that the subset $\mathrm{Y}$ of $\mathrm{X}$ is the relevant set for $\{x, y\}$ if $Y$ is sufficient for $\{x, y\}$ and every sufficient set for $\{x, y\}$ contains $Y$.

When $\mathrm{X}$ is infinite there are well defined social welfare functions for which no pair has a relevant set. There are two extremes within the family of rules for which every pair does have a relevant set: If $\mathrm{f}$ is constant (i.e., $f(p)=f(q) \forall p, q \in \mathcal{P}$ ) then the empty set is the relevant set for every pair. For some social welfare functions - the Borda rule for instance — the set $\mathrm{X}$ itself is the relevant set for every pair.

On the domain $\mathrm{L}(\mathrm{X})^{\mathrm{N}}$ the family of sets that are sufficient for a particular pair is closed under finite intersection (Campbell and Kelly, 2000):

\section{Intersection principle for the domain $\mathrm{L}(\mathrm{X})^{\mathrm{N}}$ :}

If $\mathrm{Y}$ and $\mathrm{Z}$ are each sufficient for $\{\mathrm{x}, \mathrm{y}\}$ then so is $\mathrm{Y} \cap \mathrm{Z}$.

See the remark following the proof of our theorem for other domains on which the intersection principle holds. ${ }^{3}$

If $\mathrm{X}$ is finite then every pair from $\mathrm{X}$ has a relevant set: By the intersection principle, the relevant set for $\{x, y\}$ is the intersection of all the sufficient sets for $\{x, y\}$. When $X$ is infinite there are social welfare functions for which relevant sets do not exist:

Example 1: For any profile $\mathrm{p} \in \mathrm{L}(\mathrm{X})^{\mathrm{N}}$ and any $\mathrm{x}$ and $\mathrm{y}$ in $\mathrm{X}$ set $\mathrm{x} \succ_{\mathrm{f}(\mathrm{p})} \mathrm{y}$ if $\mathrm{x} \succ_{1}^{\mathrm{p}} \mathrm{y}$ unless (i) $\mathrm{y} \succ_{2}^{\mathrm{p}} \mathrm{x}$ and (ii) there are infinitely many alternatives between $y$ and $x$ in $p(2)$ in which case set $y \succ_{f(p)} x$. It is easy to

3. For arbitrary pair $\{x, y\}$, Blau (1971) required every $\beta$-element superset of $\{x, y\}$ to be sufficient for $\{x, y\}$. Then he showed that his condition implies IIA on an unrestricted domain. (For any $\beta$ $>2$, every pair of alternatives is the intersection of all the $\beta$-element subsets of $X$ that contain that pair.) We merely posit that there is some $\beta$-element subset of $X$ that is sufficient for $\{x, y\}$. 
confirm that $\mathrm{f}$ is transitive-valued, satisfies Pareto and non-dictatorship, and treats alternatives symmetrically. It is also easy to check that $\mathrm{Y} \subset \mathrm{X}$ is sufficient for a pair $\{\mathrm{x}, \mathrm{y}\}$ if and only $\{\mathrm{x}, \mathrm{y}\} \subset \mathrm{Y}$ and $\mathrm{X} \backslash \mathrm{Y}$ is finite. Therefore, there is no relevant set for any pair: If $\mathrm{Y}$ is sufficient for $\{\mathrm{x}, \mathrm{y}\}$ then so is $Y \backslash\{z\}$ for any $z \in Y$. The set $\{x, y\}$ is the intersection of all sufficient sets for $\{x, y\}$, but $\{x, y\}$ itself is not sufficient for $\{x, y\}$.

When $X$ is countably infinite it is easy to design social welfare functions on $L(X)^{N}$ that satisfy the Pareto criterion and anonymity (and hence are not weakly sub-dictatorial) and for which each pair has a finite relevant set:

Example 2: $\mathrm{X}=<\mathrm{X}(1), \mathrm{x}(2), \ldots>$. Let $>$ be an arbitrary strong order on $\mathrm{X}$ and choose a particular numerical representation of $>$ by setting $\mathrm{u}(\mathrm{x}(1))=1$, and proceeding inductively to assign a value to $\mathrm{u}(\mathrm{x}(2))$ that agrees with the restriction of $\succ$ to $\{\mathrm{x}(1), \mathrm{x}(2)\}$, and with $\mathrm{u}(\mathrm{x}(1), \mathrm{u}(\mathrm{x}(2)), \ldots, \mathrm{u}(\mathrm{x}(\mathrm{t}))$ already defined choose $u(x(t+1)$ so that it agrees with the restriction of $\succ$ to $\{x(1), x(2), \ldots, x(t+1)\}$. At profile $\mathrm{p}$ set $\mathrm{x}(\mathrm{s}) \succeq_{\mathrm{f}(\mathrm{p})} \mathrm{x}(\mathrm{t})$ if and only if the sum of the utility numbers assigned to $\mathrm{x}(\mathrm{s})$ over all $\mathrm{i} \in \mathrm{N}$ is at least as large as the sum of the utility numbers assigned to $x(t)$ over all $i \in N$. This rule is defined on all of $L(X)^{n}$, satisfies Pareto and anonymity, and for every pair of alternatives, the relevant set is finite: given $\mathrm{x}(\mathrm{s})$ and $\mathrm{x}(\mathrm{t})$ in $\mathrm{X}$, with $\mathrm{s}<\mathrm{t}$, the relevant set for this pair is $\{\mathrm{x}(1), \mathrm{x}(2), \ldots, \mathrm{x}(\mathrm{t})\}$. If every pair in $\mathrm{X}$ has a finite sufficient set we say the rule satisfies finite dependence.

Each pair has a finite relevant set for Example 2 but for every positive integer $\beta$, however large, there is a pair with a relevant set that has more that $\beta$ members. This appears to impose prohibitively high costs of information processing. Accordingly, we investigate rules that are uniformly bounded in the following sense:

\section{Uniformly bounded dependence:}

The social welfare function $\mathrm{f}$ satisfies uniformly bounded dependence if there exists a positive integer $\beta$ such that every pair of alternatives has a sufficient set with $\beta$ elements. (Note: For a given pair there may or may not be a sufficient set with less than $\beta$ elements.)

A social welfare function on the domain $\mathrm{L}(\mathrm{X})^{\mathrm{N}}$ has a relevance map if it satisfies uniform boundedness. That is, there is a function $\psi$ mapping pairs from $X$ into subsets of $X$ such that, for each pair $\{x, y\}$ from $X, \Psi(x, y)$ is the relevant set for $\{x, y\}$. That is a consequence of the fact that for any social welfare function on $\mathrm{L}(\mathrm{X})^{\mathrm{N}}$, if $\{\mathrm{x}, \mathrm{y}\}$ has a finite sufficient set it has a relevant set: If $\mathrm{Y}$ is sufficient for $\{x, y\}$ then, by the intersection principle, every sufficient set for $\{x, y\}$ has a sufficient subset contained in $\mathrm{Y}$. Therefore, the intersection of all sufficient sets for $\{\mathrm{x}, \mathrm{y}\}$ is equal to the intersection of 
all sufficient sets for $\{\mathrm{x}, \mathrm{y}\}$ that are contained in $\mathrm{Y}$. The latter is a finite collection, so the intersection is a sufficient set. It follows that if $\{x, y\}$ has a finite sufficient set it has a relevant set. We do indeed employing the domain $\mathrm{L}(\mathrm{X})^{\mathrm{N}}$ in this section, but in anticipation of generalizations that will be the subject of further research we simply assume the existence of a function $\sigma$ that chooses a sufficient set $\sigma(\mathrm{x}, \mathrm{y})$ with no more than $\beta$ members for arbitrary $\{x, y\}$.

For the social welfare function of Example 3 below, each pair has a relevant set, and none of the relevant sets has more than four alternatives. But the rule is sub-dictatorial. This trade-off between uniform boundedness and symmetric treatment of individuals is inevitable when $\mathrm{X}$ is infinite and the social welfare function satisfies the Pareto criterion. The following social welfare function is central to Campbell and Kelly (2000). A version of it was introduced in Gibbard, Hylland, and Weymark (1987).

Example 3 (A Gateau rule): X can be any (finite or infinite) set with at least four members. Assume an odd number $\mathrm{n}$ of individuals and the domain $\mathrm{L}(\mathrm{X})^{\mathrm{N}}$ of profiles of linear orders. To define the social welfare function begin by choosing two member $\mathrm{c}$ and $\mathrm{d}$ of $\mathrm{X}$. Given profile $\mathrm{p}$ let $\mathrm{w}$ denote the majority winner from $\{c, d\}-$ set $w=c$ if $c=d$. Let $T(p)$ be the set of all $x \in X \backslash\{w\}$ such that a majority of individuals prefer $\mathrm{x}$ to $\mathrm{w}$, and let $\mathrm{B}(\mathrm{p})$ be the set of all $\mathrm{x} \in \mathrm{X} \backslash\{\mathrm{w}\}$ such that a majority of individuals prefer $w$ to $x$. To define $f(p)$ we set $x \succ w \succ y$ for all $x \in T(p)$ and all $y \in B(p)$. Finally order the pairs from $\mathrm{T}(\mathrm{p})$ according to $\mathrm{p}(1)$ and order the pairs from $\mathrm{B}(\mathrm{p})$ according to $\mathrm{p}(2)$. The resulting social welfare function is transitive-valued, non-dictatorial, and satisfies the Pareto criterion. Note that $\{x, y, c, d\}$ is the relevant set for arbitrary pair $\{\mathrm{x}, \mathrm{y}\}$ - in particular, each pair has a relevant set - so there is a uniform bound of four for this social welfare function. Some relevant sets may have less than four alternatives. For instance, $\{x, c, d\}$ is the relevant set for $\{x, c\}$.

For the social welfare function of Example 3 one would rather play the role of person 1 than person 2, because person 1's preferences prevail at the top of the social ordering unless $\mathrm{c}$ and $\mathrm{d}$ each defeat every member of $\mathrm{X} \backslash\{\mathrm{c}, \mathrm{d}\}$ by a majority. It is possible for a sub-dictatorial rule to treat individuals symmetrically (in a limited sense) as the next example demonstrates.

Example 4 ("Egalitarian" sub-dictatorship). $\mathrm{X}$ is any infinite set and $\mathrm{N}=\{1,2, \ldots, \mathrm{n}\}$ for any $\mathrm{n}$ that is a power of two. Choose two specific alternative $\mathrm{x}$ and $\mathrm{y}$ in advance, and a function $\lambda$ from $\mathrm{L}(\{\mathrm{x}, \mathrm{y}\})^{\mathrm{N}}$ to $\mathrm{N}$ such that every $i \in N$ is the image (under $\lambda$ ) of the same number of profiles in $L(\{x, y\})^{N}$ as individual 1 . For each $\mathrm{p} \in \mathrm{L}(\mathrm{X})^{\mathrm{N}}$ set $\mathrm{f}(\mathrm{p})=\mathrm{p}(\lambda(\mathrm{p} \mid\{\mathrm{x}, \mathrm{y}\})$. That is, the social ordering at profile $\mathrm{p}$ is the ordering of the individual i selected by $\lambda$ for $\mathrm{p} \mid\{\mathrm{x}, \mathrm{y}\}$. (If $\mathrm{n}$ is a large integer that is not a power of 2 we can define $\lambda$ so that it comes close to giving an individual the same chance of determining the social ordering as any 
other person.)

Although each individual has the same chance as any other to completely determine the social ordering for Example 4, any economic application, with individual preferences restricted in the usual way and with $\mathrm{X}$ allowing for arbitrary redistribution of private goods, would deliver all of the available private goods to individual $\lambda(\mathrm{p} \mid\{\mathrm{x}, \mathrm{y}\})$ at profile $\mathrm{p}$. Even in a more restricted setting in which $\mathrm{L}(\mathrm{X})^{\mathrm{N}}$ is a plausible domain, Example 4 could be punitive. For instance, if the allocation of income tax burdens is fixed, and preferences for private goods are assumed not to change, and $\mathrm{X}$ is a (countable) set of alternative health care policies then it may be reasonable to assume that arbitrary individual i's preference scheme could be any member of $L(X)$. In that case, setting $f(p)=p(i)$ is very unfair in many cases, although it is not nearly as heinous as a dictatorial rule.

\section{Results}

We will prove that uniformly bounded dependence implies that for each positive integer $\alpha \geq 3$ there is a collection $\boldsymbol{A}$ containing an infinite number of subsets of $\mathrm{X}$ each with exactly $\alpha$ members, and such that for each $\mathrm{A} \in \boldsymbol{A}$ and each pair $\{\mathrm{x}, \mathrm{y}\}$ of members of $\mathrm{A}$ there is a sufficient set for $\{\mathrm{x}, \mathrm{y}\}$ than contains no member of A other than $\mathrm{x}$ and $\mathrm{y}$. That will allow us to use Arrow's impossibility theorem in a limited way. Arrow's theorem employs independence of irrelevant alternatives, which can be expressed by saying that for every pair of alternatives $\{a, b\}$ the set $\{a, b\}$ itself is sufficient for that pair. Now, if $A \in$ A and $\mathrm{B}$ is the union of all the $\beta$-element sufficient sets over all the pairs from A, we can confine attention to a subset of profiles for which every individual has each member of A ranking above each member of $\mathrm{X} \backslash \mathrm{A}$, and the members of $\mathrm{B} \backslash \mathrm{A}$ are ranked in a fixed way. For each pair $\{\mathrm{x}, \mathrm{y}\}$ from $\mathrm{A}$ the set $\{x, y\} \cup B$ is sufficient for $\{x, y\}$. Therefore, within the specified subset of profiles, IIA holds sway overs pairs of alternatives from A. Arrow's Theorem then implies that there is an individual $\mathrm{j}$ whose preferences determine the social ordering within A and the specified subset of profiles. Consequently, $\mathrm{f}$ is sub-dictatorial. (For all of the domains considered in this paper the Pareto criterion implies that $\mathrm{x}$ and $\mathrm{y}$ both belong to every sufficient set for $\{\mathrm{x}, \mathrm{y}\}$.)

The key to our theorem is a result from graph theory: Given a non-empty set $\mathrm{V} \subset \mathrm{X}$ (of vertices) we consider the set $\mathrm{E}$ of unordered pairs $\{\mathrm{x}, \mathrm{y}\}$ of elements of $\mathrm{V}$. (Each member of $\mathrm{E}$ is called an edge of V.) A two-coloring of $E$ is a pair $\{R, B\}$ of disjoint subsets of $E$ such that $R \cup B=E$ and each member of $\mathrm{R}$ is a red edge and each member of $\mathrm{B}$ is a blue edge. (One of the sets may be empty.) A subset $\mathrm{S}$ of $\mathrm{V}$ is monochromatic if all the pairs $\{\mathrm{x}, \mathrm{y}\}$ contained in $\mathrm{S}$ are in $\mathrm{R}$ or else they are all in B. Frank $\mathrm{P}$. 
Ramsey proved the following:

Ramsey's Theorem:

If $\mathrm{V}$ is infinite then any two-coloring of the edges of $\mathrm{V}$ contains an infinite monochromatic subset. (See Bollobás, 1998, p. 186.)

Given a positive integer $\beta$, we will examine an arbitrary social welfare function $\mathrm{f}$ such that for

every pair $\{x, y\}$ of distinct alternatives in $X$ there is a sufficient subset $\sigma(x, y)$ for $\{x, y\}$ that has no more than $\beta$ members. (We streamline the notation by writing $\sigma(x, y)$ instead of $\sigma(\{x, y\})$ ). Let $\Pi$ denote the set of all pairs from $X$. The following definition and lemma can refer to any correspondence from $\Pi$ into $\mathrm{X}$, but they will be applied to a map $\sigma$ that selects sufficient sets for a given social welfare function.

\section{Fragmented set:}

Given a correspondence $\sigma: \Pi \rightarrow X$ we say that the subset $A$ of $X$ is fragmented (with respect to $\sigma)$ if we have $\sigma(\pi) \cap \mathrm{A}=\pi$ for all pairs $\pi$ from $\mathrm{A}$.

\section{Lemma :}

$\mathrm{X}$ is infinite. For all pairs of integers $(\alpha, \beta)$, with $\alpha$ and $\beta$ both greater than 1 , and any correspondence $\sigma: \Pi \rightarrow X$ such that $|\sigma(\pi)| \leq \beta$ for each $\pi \in \Pi$, there is an infinitely large collection of (pairwise disjoint) fragmented sets $\mathrm{A} \subset \mathrm{X}$ (with respect to $\sigma$ ) such that $|\mathrm{A}|=\alpha$.

Before proving this we highlight the importance of uniform boundedness by means of two examples.

Example 5: $\mathrm{X}$ is the set of positive integers. If $\mathrm{s}<\mathrm{t}$ set $\sigma(\mathrm{t}, \mathrm{s})=\sigma(\mathrm{s}, \mathrm{t})=\{\mathrm{s}, \mathrm{s}+1, \mathrm{~s}+2, \ldots, \mathrm{t}-1, \mathrm{t}\}$. Then each $\sigma(\mathrm{s}, \mathrm{t})$ is finite, but the images of $\sigma$ are not uniformly bounded. If $\mathrm{A}$ is a finite subset of $\mathrm{X}$ with at least three members let $s_{A}$ be the smallest member of $A$ and let $t_{A}$ be the largest member of $A$. Then $\sigma\left(s_{A}, t_{A}\right) \cap A=A$. Therefore, the only fragmented sets are those with only two elements.

Example 6: $\mathrm{X}$ is the set of positive integers and $\beta$ is a given positive integer. If $\mathrm{s}<\mathrm{t}$ we set $\sigma(\mathrm{t}, \mathrm{s})=$ $\sigma(s, t)=\{s, t, t+1, t+2, \ldots, t+\beta-2\}$, a set containing exactly $\beta$ members. For any positive integer $\alpha$, any set of the form $A=\{s, s+k, s+2 k, s+3 k, \ldots, s+(\alpha-1) k\}$ is fragmented if $k>\beta-1$, where $s$ is an arbitrary positive integer.

Proof of the Lemma: We will prove the claim by induction on $\beta$.

Basis Step 1. If $\beta=2$ then $\sigma(\pi)=\pi$ and every subset of $X$ is fragmented. In particular for $\alpha \geq 2$, each of the infinitely many sets A with $|\mathrm{A}|=\alpha$ is fragmented, and thus our claim is true for $(\alpha, 2)$, for all 
$\operatorname{such} \alpha$.

Induction step 1. We now establish the claim for $\beta>2$, assuming that it is true for all integers $\beta^{*}$ such that $2 \leq \beta^{*}<\beta$.

This part of the proof proceeds by induction on $\alpha$.

Basis Step 2. Every two-element subset of $X$ is fragmented, and thus there is an infinitely large collection of pairwise disjoint fragmented sets of cardinality two and our claim holds for $(2, \beta)$ for all $\beta$.

Induction Step 2. So now we suppose that for all pairs of integers $\left(\alpha^{*}, \beta^{*}\right)$ where either $\beta^{*}<\beta$ or both $\beta^{*}=\beta$ and $\alpha^{*}<\alpha$, then if $X$ is infinite, and given a correspondence $\sigma: \Pi_{X} \rightarrow X$ such that $\pi \subseteq \sigma(\pi)$ and $|\sigma(\pi)| \leq \beta^{*}$ for each $\pi \in \Pi_{X}$, there is an infinitely large collection of pairwise disjoint fragmented sets $\mathrm{A} \subset \mathrm{X}$ (with respect to $\sigma$ ) such that $|\mathrm{A}|=\alpha^{*}$. We prove the claim for $(\alpha, \beta)$.

The heart of the proof consists of showing there is at least one such fragmented set A. The existence of an infinitely large collection of pairwise disjoint fragmented sets is a consequence of the following argument. Let $\mathrm{X}_{2}=\mathrm{X} \backslash \mathrm{A}$ and $\sigma_{2}: \Pi_{2} \rightarrow \mathrm{X}_{2}$ is given by $\sigma_{2}(\mathrm{x}, \mathrm{y})=\sigma(\mathrm{x}, \mathrm{y}) \backslash \mathrm{A}$. Then $\pi \subseteq \sigma_{2}(\pi)$ and $\left|\sigma_{2}(\pi)\right| \leq \beta$ for each $\pi \in \Pi_{2}$. So applying the argument used to show the existence of one fragmented set establishes that there is an $A_{2} \subseteq X_{2}$ that is fragmented with respect to $\sigma_{2}$ for which $\left|A_{2}\right|=\alpha$. But clearly, $A_{2}$ is also fragmented with respect to $\sigma$. The argument can be used again to find an $A_{3}$ in $X_{3}=X \backslash(A \cup$ $\mathrm{A}_{2}$ ) and so on.

Now, for $\mathrm{a} \in \mathrm{X}$, set $\mathrm{V}_{\mathrm{a}}=\mathrm{X} \backslash\{\mathrm{a}\}$. Partition the edges of $\mathrm{V}_{\mathrm{a}}$ into $\mathrm{R}$ and $\mathrm{B}$, where

$$
\begin{aligned}
& \mathrm{R}=\left\{\{\mathrm{x}, \mathrm{y}\}: \mathrm{x}, \mathrm{y} \in \mathrm{V}_{\mathrm{a}}, \mathrm{x} \neq \mathrm{y} \text {, and } \mathrm{a} \in \sigma(\mathrm{x}, \mathrm{y})\right\} \text {, and } \\
& \mathrm{B}=\left\{\{\mathrm{x}, \mathrm{y}\}: \mathrm{x}, \mathrm{y} \in \mathrm{V}_{\mathrm{a}}, \mathrm{x} \neq \mathrm{y} \text {, and } \mathrm{a} \notin \sigma(\mathrm{x}, \mathrm{y})\right\} .
\end{aligned}
$$

By Ramsey's Theorem, $\mathrm{V}_{\mathrm{a}}$ contains an infinite monochromatic subset $\mathrm{X}^{\prime}$.

Case 1: For at least one a in $X$ the set $V_{a}$ has an infinite subset $X^{\prime} \subset X \backslash\{a\}$ all of whose edges are in $R$. Define the correspondence $\sigma^{\prime}$ by setting $\sigma^{\prime}(\mathrm{x}, \mathrm{y})=\sigma(\mathrm{x}, \mathrm{y}) \backslash\{\mathrm{a}\}$ for all distinct $\mathrm{x}$ and $\mathrm{y}$ in $\mathrm{X}^{\prime}$. Because a belongs to $\sigma(\mathrm{x}, \mathrm{y})$ the set $\sigma^{\prime}(\mathrm{x}, \mathrm{y})$ has $\beta-1$ members for all $\mathrm{x}, \mathrm{y}$ in $\mathrm{X}^{\prime}$. Then by the induction hypothesis on $\beta$, for each integer $\alpha \geq 2$ the mapping $\sigma^{\prime}$ has an infinite number of fragmented sets in $X^{\prime}$ with exactly $\alpha$ members. Let $\mathrm{A} \subset \mathrm{X}^{\prime}$ be any such fragmented set. Because a does not belong to $\mathrm{X}^{\prime}$ it does not belong to $A$, and thus for every pair $\{x, y\}$ from A the intersection of $\sigma^{\prime}(x, y) \cup\{a\}=\sigma(x, y)$ with $A$ is $\{x, y\}$ itself. Thus for each integer $\alpha \geq 3$ the mapping $\sigma$ has an infinite number of fragmented sets with exactly $\alpha$ 
members.

Case 2: If Case 1 does not hold, then for every a in $X$ the set $V_{a}$ does not have an infinite subset $X^{\prime} \subset$ $\mathrm{X} \backslash\{\mathrm{a}\}$ all of whose edges are in $\mathrm{R}$. Hence $\mathrm{V}_{\mathrm{a}}$ has an infinite subset $\mathrm{X}^{\prime}$ all of whose edges are in $\mathrm{B}$ for each $a \in X$.

Select any one such alternative a. Recall that by the definition of $\mathrm{B}, \mathrm{a} \notin \sigma(\mathrm{x}, \mathrm{y})$ for all $\mathrm{x}$ and $\mathrm{y}$ in $\mathrm{X}^{\prime}$. We apply Ramsey's Theorem again, this time using $\mathrm{X}^{\prime}$ as the given set of vertices. We set

$$
\begin{aligned}
& \mathrm{R}^{\prime}=\left\{\{\mathrm{x}, \mathrm{y}\}: \mathrm{x}, \mathrm{y} \in \mathrm{X}^{\prime} \text { and } \mathrm{y} \notin \sigma(\mathrm{a}, \mathrm{x}) \text { and } \mathrm{x} \notin \sigma(\mathrm{a}, \mathrm{y}) \text { both hold }\right\} \text {, and } \\
& \mathrm{B}^{\prime}=\left\{\{\mathrm{x}, \mathrm{y}\}: \mathrm{x}, \mathrm{y} \in \mathrm{X}^{\prime} \text { and either } \mathrm{y} \in \sigma(\mathrm{a}, \mathrm{x}) \text { or } \mathrm{x} \in \sigma(\mathrm{a}, \mathrm{y})\right\} .
\end{aligned}
$$

By Ramsey's Theorem, $\mathrm{X}^{\prime}$ contains an infinite monochromatic subset. Then either there is an infinite subset $\mathrm{Y}$ of $\mathrm{X}^{\prime}$ such that $\mathrm{y} \notin \sigma(\mathrm{a}, \mathrm{x})$ and $\mathrm{x} \notin \sigma(\mathrm{a}, \mathrm{y})$ for all $\mathrm{x}$ and $\mathrm{y}$ in $\mathrm{Y}$, or there is an infinite subset $\mathrm{Z}$ of $\mathrm{X}^{\prime}$ such that for all $\mathrm{x}$ and $\mathrm{y}$ in $\mathrm{Z}$ either $\mathrm{y} \in \sigma(\mathrm{a}, \mathrm{x})$ and $\mathrm{x} \in \sigma(\mathrm{a}, \mathrm{y})$.

Case 2-A. Suppose that there is an infinite subset $Y$ of $X^{\prime}$ such that $\mathrm{y} \notin \sigma(\mathrm{a}, \mathrm{x})$ and $\mathrm{x} \notin \sigma(\mathrm{a}, \mathrm{y})$ for all $\mathrm{x}$ and $\mathrm{y}$ in $\mathrm{Y}$. Because $\mathrm{Y}$ is infinite it contains a fragmented set $\mathrm{A}$ with exactly $\alpha-1$ members. We show that $\mathrm{A} \cup\{\mathrm{a}\}$ is fragmented. Because $\mathrm{Y}$ is a subset of $\mathrm{X}^{\prime}$ we have $\mathrm{a} \notin \sigma(\mathrm{x}, \mathrm{y})$ for all $\mathrm{x}$ and $\mathrm{y}$ in $\mathrm{Y}$. Because $\mathrm{A}$ is fragmented we have $\mathrm{z} \notin \sigma(\mathrm{x}, \mathrm{y})$ for any three distinct members $\mathrm{x}, \mathrm{y}$, and $\mathrm{z}$ of $\mathrm{A}$. Finally, because $\mathrm{A}$ is a subset of $\mathrm{Y}$ we have $\mathrm{x} \notin \sigma(\mathrm{a}, \mathrm{y})$ for any two distinct members $\mathrm{x}$ and $\mathrm{y}$ of $\mathrm{A}$. Therefore, $\mathrm{A} \cup\{\mathrm{a}\}$ is a fragmented set with $\alpha$ members.

Case 2-B. Suppose that there is an infinite subset $\mathrm{Z}$ of $\mathrm{X}^{\prime}$ such that for all $\mathrm{x}$ and $\mathrm{y}$ in $\mathrm{Z}$ either $\mathrm{y} \in \sigma(\mathrm{a}, \mathrm{x})$ or $\mathrm{x} \in \sigma(\mathrm{a}, \mathrm{y})$. It follows that for every $\mathrm{x} \in \mathrm{Z}$ we have $\mathrm{x} \in \sigma(\mathrm{a}, \mathrm{y})$ for all $\mathrm{y} \in \mathrm{Z} \backslash \sigma(\mathrm{a}, \mathrm{x})$. Choose $\beta-2$ distinct members $x_{1} x_{2} \ldots, x_{\beta-2}$ of $Z$. Let

$$
Z^{\prime}=\left\{y \in Z: y \notin \sigma\left(a, x_{t}\right) \text { for } t=1,2, \ldots, \beta-2\right\}
$$

Then $Z^{\prime}$ is infinite because the images of $\sigma$ are finite. Because $y \notin \sigma\left(a, x_{t}\right)$ for $y \in Z^{\prime}$ we have $x_{t} \in \sigma(a, y)$ for all $y \in Z^{\prime}$ and all $t=1,2, \ldots, \beta-2$. Because $|\sigma(\pi)| \leq \beta$ for each two-element subset $\pi$ of $X$ we have

$$
\sigma(\mathrm{a}, \mathrm{y})=\left\{\mathrm{a}, \mathrm{y}, \mathrm{x}_{1} \mathrm{x}_{2} \ldots, \mathrm{x}_{\beta-2}\right\} \text { for all } \mathrm{y} \in \mathrm{Z}^{\prime} .
$$

By the induction hypothesis the infinite set $Z^{\prime}$ has a fragmented set $A$ with exactly $\alpha-1$ members. We conclude by showing that $\mathrm{A} \cup\{\mathrm{a}\}$ is fragmented: We have $\mathrm{X}_{\beta-2} \subset \mathrm{X}^{\prime}$ and thus $\mathrm{a} \notin \sigma(\mathrm{x}, \mathrm{y})$ for any two $\mathrm{x}$ and $\mathrm{y}$ in A. Because A is fragmented we have $\mathrm{z} \notin \sigma(\mathrm{x}, \mathrm{y})$ for any three distinct members of A. Finally, 
because $\sigma(\mathrm{a}, \mathrm{y})=\left\{\mathrm{a}, \mathrm{y}, \mathrm{x}_{\mathrm{t}} \mathrm{x}_{\mathrm{t}} \ldots, \mathrm{x}_{\beta-2}\right\}$ for all distinct $\mathrm{y}$ and $\mathrm{z}$ in $\mathrm{A}$ we have $\mathrm{z} \notin \sigma(\mathrm{a}, \mathrm{y})$. This establishes that $\mathrm{A} \cup\{\mathrm{a}\}$ is fragmented.

From now on when we employ the function $\sigma$ it will be understood that $\sigma$ specifies some sufficient set (for the given social welfare function) for each pair of alternatives. Uniform boundedness on any domain that contains $\mathrm{L}(\mathrm{X})^{\mathrm{N}}$ implies that each pair $\{\mathrm{x}, \mathrm{y}\}$ has a relevant set, so we could let $\sigma(\mathrm{x}, \mathrm{y})$ be that relevant set.

\section{Theorem:}

$X$ is any infinite set and $\rho=W(X)^{N}$ or $L(X)^{N}$. If $f: \rho \rightarrow W(X)$ satisfies the Pareto criterion and uniformly bounded dependence then it is sub-dictatorial.

Proof: Given a transitive-valued social welfare function $\mathrm{f}$ satisfying Pareto and uniformly bounded dependence, for each pair $\{x, y\}$ from $X$ let $\sigma$ choose a sufficient set $\sigma(x, y)$ with no more that $\beta$ members, where $\beta$ is some positive integer with respect to which $\mathrm{f}$ satisfies uniform boundedness. Let $\mathrm{A}$ be any fragmented set with respect to $\sigma$. Let $\mathrm{B}$ be the union of the sets $\sigma(\mathrm{x}, \mathrm{y}) \backslash\{\mathrm{x}, \mathrm{y}\}$ over all distinct $\mathrm{x}$ and $\mathrm{y}$ in $\mathrm{A}$. (Note that the Pareto criterion implies that for all distinct $\mathrm{x}$ and $\mathrm{y}$ in $\mathrm{X}$ we have $\{\mathrm{x}, \mathrm{y}\} \subseteq$ $\sigma(\mathrm{x}, \mathrm{y})$.) Because $\mathrm{A}$ is fragmented, $\mathrm{A}$ and $\mathrm{B}$ are disjoint. Now we define a social welfare function $\mathrm{g}$ on domain $L(A)^{N}$. First choose any profile $r$ in $\rho$ and let $\rho^{\prime}$ be any subset of $\rho$ with the following three properties:

$$
\rho^{\prime} \mid A=W(A)^{N} \text { or } L(A)^{N} \text {. }
$$

(ii) For all $\mathrm{a} \in \mathrm{A}$ and $\mathrm{b} \in \mathrm{B}$ we have $\mathrm{p}|\{\mathrm{a}, \mathrm{b}\}=\mathrm{q}|\{\mathrm{a}, \mathrm{b}\}$ for all $\mathrm{p}$ and $\mathrm{q} \in \mathrm{P}^{\prime}$.

$$
\mathrm{p}|\mathrm{B}=\mathrm{r}| \mathrm{B} \text { for all } \mathrm{p} \in \rho^{\prime} .
$$

For any profile $s \in L(A)^{N}$ let $p^{s}$ be some profile in $\rho^{\prime}$ such that $p^{s} \mid A=s$. Suppose that a and $b$ are two distinct members of $A$ and $u$ and $v$ are two profiles in $\rho^{\prime} \mid A$ such that $u|\{a, b\}=v|\{a, b\}$. Because $\sigma(a, b) \cap A=\{a, b\}$ we have $p^{u}\left|\sigma(a, b)=p^{v}\right| \sigma(a, b)$ and hence $f\left(p^{u}\right)\left|\{a, b\}=f\left(p^{v}\right)\right|\{a, b\}$. This in turn implies that $f\left(p^{u}\right)\left|A=f\left(p^{v}\right)\right| A$ if $p^{u}\left|A=p^{v}\right| A$. Therefore, the social welfare function $g: \rho^{\prime} \mid A \rightarrow W(A)$ is well defined if we set $\mathrm{g}(\mathrm{s})=\mathrm{f}\left(\mathrm{p}^{\mathrm{s}}\right) \mid$ A. Also, $\mathrm{g}$ satisfies IIA because $\mathrm{u}|\{\mathrm{a}, \mathrm{b}\}=\mathrm{v}|\{\mathrm{a}, \mathrm{b}\}$ implies $\mathrm{p}^{\mathrm{u}} \mid \sigma(\mathrm{a}, \mathrm{b})=$ $\mathrm{p}^{\mathrm{v}} \mid \sigma(\mathrm{a}, \mathrm{b})$ which in turn implies $\mathrm{f}\left(\mathrm{p}^{\mathrm{u}}\right)\left|\{\mathrm{a}, \mathrm{b}\}=\mathrm{f}\left(\mathrm{p}^{\mathrm{v}}\right)\right|\{\mathrm{a}, \mathrm{b}\}$. And g satisfies the Pareto criterion because $\mathrm{f}$ does. It follows from Arrow's impossibility theorem that $\mathrm{g}$ is dictatorial, and hence $\mathrm{f}$ is sub-dictatorial.

Remark: The following domain condition is key to establishing the existence of relevant sets, but we 
emphasize that our theorem is valid on many domains for which the existence of relevant sets cannot be guaranteed because we only used the fact that $\sigma(\pi)$ is sufficient for $\pi$.

\section{Well structured domain:}

We say that $\rho$ is a well structured domain if, for any two subsets $Y$ and $Z$ of $X$, for any $p, q \in \rho$ such that $\mathrm{p}|\mathrm{Y} \cap \mathrm{Z}=\mathrm{q}| \mathrm{Y} \cap \mathrm{Z}$ there is a profile $\mathrm{r} \in \rho$ such that $\mathrm{r}|\mathrm{Y}=\mathrm{p}| \mathrm{Y}$ and $\mathrm{r}|\mathrm{Z}=\mathrm{q}| \mathrm{Z}$.

Both $\mathrm{W}(\mathrm{X})^{\mathrm{N}}$ and $\mathrm{L}(\mathrm{X})^{\mathrm{N}}$ are well structured domains of course. Let $\mathrm{M}(\mathrm{X})$ denote the set of orderings that are order-isomorphic to the "less than" ordering of the positive integers. In other words a strong ordering $\succeq$ belongs to $\mathrm{M}(\mathrm{X})$ if and only if every subset $\mathrm{Y}$ of $\mathrm{X}$ contains an alternative $\mathrm{y}$ such that $\mathrm{y} \succ \mathrm{x}$ for all $\mathrm{x} \in$ $\mathrm{Y} \backslash\{\mathrm{y}\}$ Then $\mathrm{M}(\mathrm{X})^{\mathrm{N}}$ is well structured, as is $\mathrm{O}(\mathrm{X})^{\mathrm{N}}$, where $\mathrm{O}(\mathrm{X})=\{\mathrm{R} \in \mathrm{L}(\mathrm{X}): \mathrm{R}$ is the opposite (or inverse) of some $R^{\prime}$ in $\left.M(X)\right\} . B(X)^{N}$ is well structured, where $B(X)=\{R \in L(X):$ for all $x, y \in X$ the set $\{z \in X: x \succ z \succ y\}$ is finite, where $\succ$ is the asymmetric factor of $R\}$. (The domain $B(X)^{N}$ can be used to extend the Borda rule to countably infinite sets of alternatives. See Example 2 in Campbell and Kelly, 2006.) Any singleton domain is well structured.

\section{Intersection principle for a well structured domain:}

If $\mathrm{Y}$ and $\mathrm{Z}$ are each sufficient for $\{\mathrm{x}, \mathrm{y}\}$ then so is $\mathrm{Y} \cap \mathrm{Z}$.

The intersection principle follows from the definitions of sufficient set and well structured domain: If $\mathrm{p}|\mathrm{Y} \cap \mathrm{Z}=\mathrm{q}| \mathrm{Y} \cap \mathrm{Z}$ and $\mathrm{Y}$ and $\mathrm{Z}$ are each sufficient for $\{\mathrm{x}, \mathrm{y}\}$ then $\mathrm{p}|\mathrm{Y}=\mathrm{r}| \mathrm{Y}$ implies $\mathrm{f}(\mathrm{p}) \mid\{\mathrm{x}, \mathrm{y}\}=$ $\mathrm{f}(\mathrm{r}) \mid\{\mathrm{x}, \mathrm{y}\}$ and $\mathrm{r}|\mathrm{Z}=\mathrm{q}| \mathrm{Z}$ implies $\mathrm{f}(\mathrm{r})|\{\mathrm{x}, \mathrm{y}\}=\mathrm{f}(\mathrm{q})|\{\mathrm{x}, \mathrm{y}\}$.

\section{Conclusion}

There are important statements in mathematics — and especially in mathematical economics — that are true when the number two is substituted for a key parameter, but false for any larger integer.

Consider the statement "If $X$ has at least three members and $\beta$ is the least upper bound on the cardinality of the set of alternatives over which individual preference must be consulted so that a pair of alternatives can be socially ordered, then there is no transitive-valued social welfare function on $L(X)^{\mathrm{N}}$ satisfying Pareto and non-dictatorship." Arrow proved that this statement is true if $\beta=2$. Example 3 shows that it is false when $\beta$ is greater than 2 . However, this paper proves that when the bound is greater than two there are many — an infinite number, in fact — of situations in which a single individual has far too much power. 
The theorem proved above raises a number of questions. For instance, does the result extend to situations in which $\mathrm{X}$ is a connected topological space and the domain includes only individual preferences that are continuous in that topology? We will investigate this question in a companion paper. 


\section{References}

Arrow, K. J. (1963) Social Choice and Individual Values (2nd ed., New York: Wiley).

Baumol, William (1952) Review of Social Choice and Individual Values. Econometrica 20: 110-111.

Blau, J. H. (1971) Arrow's theorem with weak independence. Economica 38: 413-420.

Bollobás, B. (1998) Modern Graph Theory. New York: Springer-Verlag.

Campbell, D. E. (1992) Equity, Efficiency, and Social Choice. Oxford: The Clarendon Press.

Campbell, D. E. and J. S. Kelly (1993) t or 1-t. That is the trade-off. Econometrica 61: 1355-1365.

Campbell, D. E. and J. S. Kelly (2000) Information and preference aggregation. Social Choice and Welfare 17: 85-93.

Campbell, D. E. and J. S. Kelly (2002) Impossibility theorems in the Arrovian framework. Handbook of Social Choice and Welfare, Volume 1. K.J. Arrow, A. K. Sen, and K. Suzumura, eds. pages 35-94. New York: Elsevier.

Campbell, D. E. and J. S. Kelly (2007) Social welfare functions that satisfy Pareto, anonymity, and neutrality, but not IIA: Countably Many Alternatives. In The Mathematics of Preference, Choice, and Order: Essays in Honor of Peter C. Fishburn. Edited by SJ Brams, WV Gehrlein and FS Roberts. Berlin: Springer, forthcoming.

Cowell, Frank (2006). Microeconomics: Principle and Analysis. Oxford: Oxford University Press.

Fleurbaey, Marc (2003) On the informational basis of social choice. Social Choice and Welfare 21: 347-384.

Fleurbaey, Marc, K. Suzumura, and K. Tadenuma (2005) Arrovian aggregation in economic environments: How much should we know about indifference surfaces?" Journal of Economic Theory 124: 22-44.

Fleurbaey, Marc, and F. Maniquet (2005) Fair social orderings when agents have unequal production skills. Social Choice and Welfare 24: 93-128.

Gibbard, A., A. Hylland, and J. A. Weymark (1987) Arrow's theorem with a fixed feasible alternative. Social Choice and Welfare 4: 105-115.

Osborne, Dale K. (1976) Irrelevant Alternatives and Social Welfare. Econometrica 44: 1001-1015.

Sen, A. K. (1986) Social Choice Theory. Handbook of Mathematical Economics, Volume III. K.J. Arrow and M. D. Intrilligator, eds. pages 1073-1181. New York: Elsevier. 